\title{
Gaphene-Oxide Based Fluorescent DNA Aptasensor for Liver Cancer Diagnosis and Therapy
}

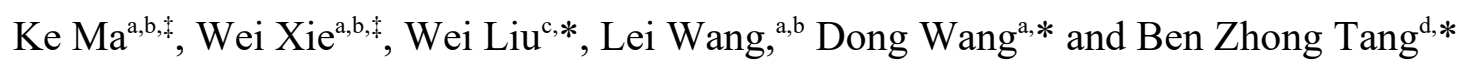

${ }^{a}$ Center for AIE Research, Shenzhen Key Laboratory of Polymer Science and Technology, Guangdong Research Center for Interfacial Engineering of Functional Materials, College of Materials Science and Engineering, Shenzhen University, Shenzhen 518060, China

${ }^{\mathrm{b}}$ Key Laboratory of Optoelectronic Devices and Systems of Ministry of Education and Guangdong Province, College of Optoelectronic Engineering, Shenzhen University, Shenzhen 518060, China

'Key Laboratory of Artificial Micro- and Nano- Structures of Ministry of Education, School of Physics and Technology, Wuhan University, Wuhan 430072, China

${ }^{\mathrm{d} H o n g}$ Kong Branch of Chinese National Engineering Research, Center for Tissue Restoration and Reconstruction, Department of Chemistry, The Hong Kong University of Science and Technology, Clear Water Bay, Kowloon, Hong Kong 999077, China 
ABSTRACT: Graphene-oxide (GO) based fluorescent DNA aptasensors are promising nanomaterials in bioassays due to the fluorescent ultra-sensitivity and target identification ability. However, the in vivo application remains an appealing yet significantly challenging task, as those developed DNA aptamers cannot across the cell membrane. In this contribution, we demonstrate for the first time such a nanomaterial for in vivo diagnosis and therapy of liver tumor. A DNA nanomaterial comprising of DNA tetrahedron and aptamers, aggregation-induced emission luminogen (AIEgens) and antitumor drug doxorubicin, is fabricated and attached on GO surface. This developed hybrid with good biocompatibility exhibits high selectivity to target liver cancer cells, and well performs for in vitro and in vivo liver tumor fluorescence imaging diagnosis and chemotherapy. Additionally, a GO-based fluorescent DNA nanodevice was also constructed by using microfluidic chip for liver tumor cell screening.

KEYWORDS: DNA aptamer, DNA tetrahedron, aggregation-induced emission, cell screening, cancer theranostics

Graphene-oxide (GO) based fluorescent DNA aptasensors are extensively used in biomedical sensing due to their intrinsic advantages, such as excellent sensitivity, high selectivity, good biocompatibility and programmability. ${ }^{1-2}$ Fluorescent DNA aptasensors are consisted of DNA aptamers and fluorophores mainly through two interaction modes, including chemically covalent bond and supramolecular interaction. ${ }^{3-5}$. Compared with the former strategy, fluorophoreunlabeled supramolecular interaction is highly desired for sensing applications thanks to the much simpler synthesis and purification procedures. ${ }^{6-7}$ To enhance the sensitivity and decline the background noise of fluorescent DNA aptasensors, GO is usually utilized as fluorescent 
quenching material. ${ }^{8-9}$ GO can easily bind fluorophores through electrostatic interactions and hydrophobic interactions, thus efficiently quenches the fluorescence by FRET (Förster resonance energy transfer). ${ }^{10}$ GO can also absorb DNA aptamers via hydrogen bonds and $\pi$-stacking interactions. ${ }^{11}$ After recognizing and interacting with target analytes, fluorescent DNA aptamer will escape from GO surface and recover its fluorescence, indicating a simple "mix and go" protocol, which is supremely powerful for in vitro sensing. ${ }^{12-13}$ To the best of our knowledge, GO based label-free fluorescent DNA aptasensors, however, have not been applied for in vivo applications, as those involved DNA aptamers are easily hydrolyzed, as well as the heavy nonspecific desorption of nontarget molecules from GO. ${ }^{14}$

Given the circumstances, the development of DNA aptamers that are unable to be hydrolyzed would be significantly important. DNA tetrahedrons (DNA-tetra), ${ }^{15-16}$ consisting of four ssDNA sequences by complementary self-assembly, have been recognized as an effective drug carrier for intracellular imaging and tumor treatment, benefiting from that DNA-tetra can rapidly across the cell membrane through caveolin-dependent pathway without being hydrolyzed. ${ }^{17-18}$ Although aptamer modified DNA-tetra has been recently utilized for fluorescent in vitro and in vivo applications, ${ }^{19-21}$ the targeting selectivity and signal-to-noise ratio remain to be further improved. To this end, locating of fluorescent DNA-tetra on GO surface could be a brilliant approach to achieve in vivo diagnosis and treatment.

In this paper, we develop for the first time a GO-based fluorescent DNA nanomaterial for in vivo diagnosis and therapy of liver tumor cells. As illustrated in Scheme 1, four ssDNA sequences self-assemble to a DNA-tetra architecture, which is modified with three hairpin switch aptamers for recognizing the target cell. Doxorubicin (DOX), a reputable antitumor drug, is loaded in the DNA-tetra skeleton through intercalation. DOX is a well-known aggregation- 
caused quench dye, which has a fluorescence quenching effect when loaded in the DNA-tetra skeleton. In order to enhance DOX fluorescence, DSAI, a fluorescent probe with typical aggregation-induced emission (AIE) features ${ }^{22}$ is also loaded in the DNA-tetra structure mainly via intercalation, presenting green fluorescence. FRET process proceeds from DSAI to DOX, which efficiently enhance the emission intensity of DOX, resulting in bright red fluorescence of the fabricated DNA nanomaterial. With the aid of the hydrogen bond interaction between the hairpin switch aptamers and GO, the DNA nanomaterial is immobilized on GO surface, leading to vanished fluorescence intensity. This presented nanomaterial is able to selectively target liver tumor cells with assistance of the hairpin structure of aptamers, and the aptamers accompanied DNA nanomaterial then escape from GO surface. Both in vitro and in vivo evaluations show that this developed protocol is effective in cancer theranostics. Moreover, a GO-based fluorescent DNA nanodevice on the basis of polydimethylsiloxane (PDMS) microfluidic chip is constructed and utilized for liver tumor cell screening. 

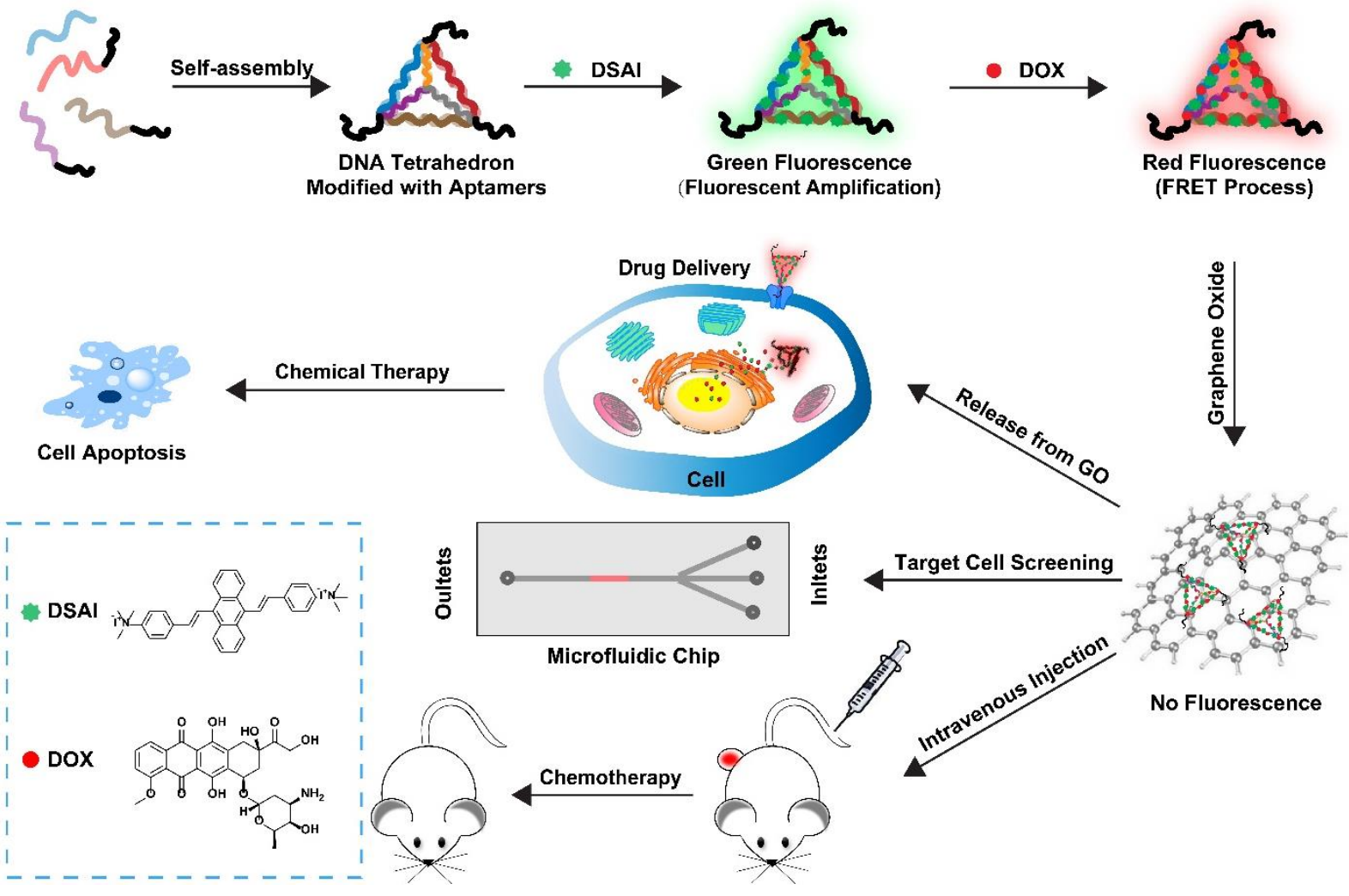

Scheme 1. Illustration of the "mix and go" GO-based fluorescent DNA nanomaterial for diagnosis and treatment of liver tumor cells (7721 cells).

\section{RESULTS AND DISCUSSION}

Three different DNA-tetras were prepared by using different DNA sequences to testify the formation of DNA-tetra (Figure S1A, S1B). As shown in Figure S1C, the electrophoretic band of DNA-1 containing one DNA aptamer sequence ${ }^{23}$ was below $200 \mathrm{bp}$, and that of DNA-3 containing three DNA aptamer sequences was above $200 \mathrm{bp}$. All the three DNA-tetras ran slowly comparing with P2 (ssDNA, $106 \mathrm{bp}$ ), verifying the formation of DNA-tetras. The morphology of DNA-3 was measured by AFM image (Figure S1D). The diameter of DNA-3 was determined to be in the range from 10 to $20 \mathrm{~nm}$. With a suitable size for cellular endocytosis, DNA-3 was indeed a desirable drug carrier for tumor therapy. 
To figure out the optimal drug proportion in DNA-3, we investigated the contents of $\mathrm{DSAI}^{24}$ and DOX in DNA-3 by fluorescence measurement, respectively. As displayed in Figure 1A, low concentration of DSAI $(0.1,0.5,1.0 \mu \mathrm{M})$ presented no emission in water solution, but high concentration of DSAI $(5-50 \mu \mathrm{M})$ exhibited a rather high fluorescence background in water. Thus 1.0 $\mu \mathrm{M}$ DSAI was chosen as the optimal DSAI content in the DNA nanomaterial. DNA-3 was then added into DSAI $(1.0 \mu \mathrm{M})$ solution to light up the emission of DSAI (Figure 1B). After the addition of $100 \mathrm{nM}$ DNA-3, the fluorescence intensity was quite high, and further addition of DNA-3 $(250 \mathrm{nM})$ could not continuously enhance the DSAI fluorescence. We also added different concentrations of DSAI into $100 \mathrm{nM}$ DNA-3 solution to evaluate the loading capacity of DNA-3 (Figure 1C). With the increasing amount of DSAI $(0.1-10 \mu \mathrm{M})$, the fluorescence of DSAI gradually grew to the maximum, indicating that the DSAI loading capacity of DNA-3 was about $10 \mu \mathrm{M}$. Further increasing amount of DSAI would cause fluorescence quenching, probably due to the aggregates sediment appearing in the mixture. In order to load high content of DOX in DNA-3, we chose the mixture of $1.0 \mu \mathrm{M}$ DSAI and $100 \mathrm{nM}$ DNA-3 for the following experiments. As depicted in Figure 1D, there was an obvious FRET outcome between DSAI and DOX. With the increasing the amount of DOX, the DSAI emission at $519 \mathrm{~nm}$ was quenched and the DOX emission at 558 and $593 \mathrm{~nm}$ was enhanced by FRET process. When DOX concentration reached $20 \mu \mathrm{M}$, the fluorescence intensity reached the maximum, and further addition of DOX $(30-40 \mu \mathrm{M})$ would quench the fluorescence of DOX. It indicated that the DOX loading capacity of DSAI/DNA-3 $(1.0 \mu \mathrm{M} / 100 \mathrm{nM})$ mixture was $20 \mu \mathrm{M}$. After confirming the optimal drug proportion in DNA nanomaterial, different concentrations of GO were added in the DNA nanomaterial to attach the DNA-3 and quench the fluorescence. The fluorescence was 
gradually quenched by the increasing amount of GO (Figure 1E). $40 \mu \mathrm{g} / \mathrm{mL}$ GO was enough to immobilize DNA-3 and quench the fluorescence to achieve a low background.

The cytotoxicity of three cells (HepG2, LO2, 7721) could also help to confirm the optimal drug proportion. In the mixture of DSAI/DNA-3 (ratio: 10/1), the cytotoxicity was raised with the increasing concentration of DSAI from 0.25 to $1.0 \mu \mathrm{M}$ (Figure $1 \mathrm{~F}$ ). The viability of nontarget cells (HepG2 and LO2) was above 85\%, while the target 7721 cell viability was below $80 \%$. Thus, the concentration of DSAI higher than $1.0 \mu \mathrm{M}$ would cause higher cytotoxicity (viability: probably below $85 \%$ ) for non-target cells, which was not available for bioimaging. Although the higher loading capacity of DOX in DSAI/DNA-3 mixture led to lower viability for both non-target cells and target cell (Figure 1G), there was still an obvious distinction between target cell and non-target cells. Always, the viability of non-target cells was higher than that of target cell, indicating that this DNA nanomaterial could eliminate target cell more easily than non-target cells. The continuous addition of GO was capable of enhancing the selectivity of this DNA nanomaterial to target cell, decreasing the cytotoxicity for non-target cells, as well as showing some influences to target cell (Figure 1H). When the amount of GO reached $40 \mu \mathrm{g} / \mathrm{mL}$, the viability of non-target cells was above $85 \%$, while the viability of target cell was below $15 \%$. Therefore, GO could efficiently increase the selectivity by immobilize DNA nanomaterial on surface, and only target cells could recognize DNA nanomaterial by aptamer-target protein binding, then DNA nanomaterial escaped from GO surface and marked target cells. On the contrary, non-target cells could not recognize the DNA nanomaterial, leading to the remain of DNA nanomaterial on GO surface.

To further study the morphology and potential change of DNA-3 after loading the DNA nanomaterial, DLS and Zeta potential measurements were conducted. As shown in Figure S2, the 
average diameter of DNA-3 was $13.6 \mathrm{~nm}$, and the average Zeta potential was $-5.92 \mathrm{mV}$. After loading with the optimal proportion of DSAI and DOX, the average diameter of DSAI/DOX/DNA-3 enlarged to $36.5 \mathrm{~nm}$, along with the average Zeta potential of $-9.14 \mathrm{mV}$. It was because the loading could enlarge the superficial area of DNA-3, and the enlarged superficial area had more negative surface potential, thus resulting in the decrease of Zeta potential. The naked GO possessed an average diameter of $158.3 \mathrm{~nm}$, and an average Zeta potential of $-26.73 \mathrm{mV}$. Upon loading DSAI/DOX/DNA-3 mixture to GO surface, the average diameter greatly increased to $814.9 \mathrm{~nm}$, and the average Zeta potential was determined to be $11.0 \mathrm{mV}$. It seems reasonable to infer that the aggregation occurred between $\mathrm{GO}$ and DSAI/DOX/DNA-3, which caused the multiple diameter rise. And this aggregation also decreased the exposed superficial area of GO, which resulted in the increase of Zeta potential. Despite the aggregation of GO, the average diameter of DSAI/DOX/DNA-3 (DNA nanomaterial) was below $50 \mathrm{~nm}$, which was suitable for in vivo imaging. The GO aggregates would not enter the membrane of tumor cells, only the DNA nanomaterials on GO surface could target the specific protein on tumor cell membrane, and then stained the tumor cells. 

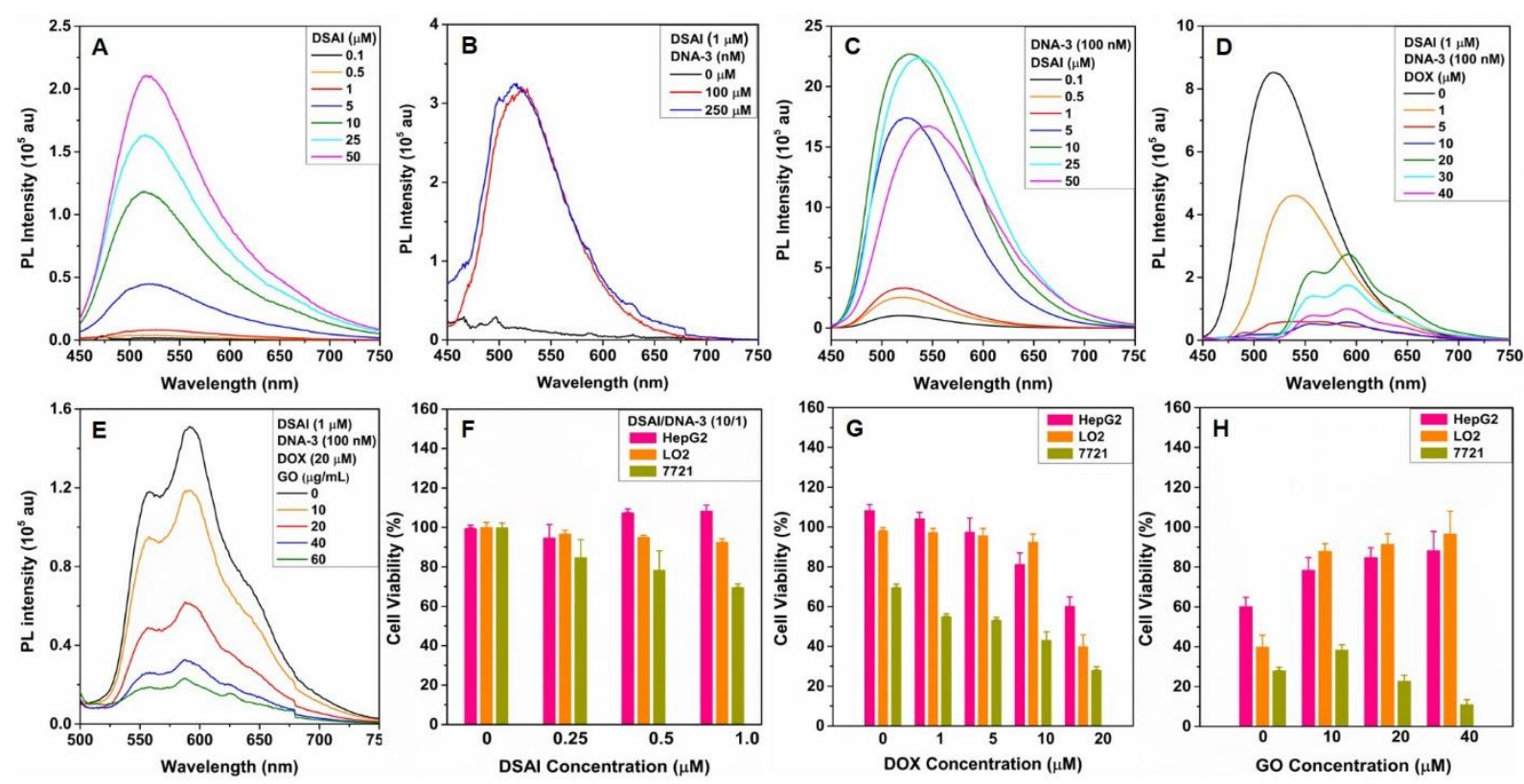

Figure 1. (A) Fluorescence spectra of different concentrations of DSAI in pure water, excitation: $405 \mathrm{~nm}$; (B) Fluorescence spectra of $1.0 \mu \mathrm{M}$ DSAI solution with different DNA-3 concentrations, excitation: $405 \mathrm{~nm}$; (C) Fluorescence spectra of DSAI with the increasing concentrations $100 \mathrm{nM}$ DNA-3 solution, excitation: $405 \mathrm{~nm}$; (D) Fluorescence spectra of different concentrations of DOX in DSAI/DNA-3 (1.0 $\mu \mathrm{M} / 100 \mathrm{nM})$ mixture, excitation: $405 \mathrm{~nm}$; (E) Fluorescence spectra of different concentrations of GO in DSAI/DOX/DNA-3 (1.0 $\mu \mathrm{M} / 20$ $\mu \mathrm{M} / 100 \mathrm{nM}$ ) mixture, excitation: $405 \mathrm{~nm}$; Viability of HepG2, LO2 and 7721 cells after stained with different concentrations of (F) DSAI/DNA-3 dye, (G) DOX loaded in DSAI/DNA-3 mixture, or $(\mathrm{H}) \mathrm{GO}$ in DSAI/DOX/DNA-3 mixture (DSAI concentration: $1.0 \mu \mathrm{M}$; DOX concentration: $20 \mu \mathrm{M}$; DNA-3 concentration: $100 \mathrm{nM}$ ).

To figure out the labeling capacity of this GO-based DNA nanomaterial, target 7721 cells were cultured with the GO-based DNA nanomaterial for different time (Figure 2). In the beginning, DSAI (green emission) and DOX (red emission) both located in cytoplasm. With the continuing staining time, a large amount of DSAI remained in cytoplasm, while DOX and small amount of 
DSAI gradually entered nucleus, thus FRET process still happened in nucleus to achieve the red emission of DOX. To ensure the accurate location of DSAI and DOX in cells, we also stained 7721 cells for 120 min with DSAI/DNA-3/GO and DOX/DNA-3/GO, respectively. With $405 \mathrm{~nm}$ excitation, DSAI/DNA-3/GO provided a strong green emission in cytoplasm and weak emission in nucleus, indicating that small amount of DSAI could enter nucleus (Figure S3A), while DOX/DNA-3/GO had no red emission under the same excitation conditions. These results indicated that the emission of DOX was indeed induced by FRET process from DSAI in the case of DSAI/DOX/DNA-3/GO (Figure S3B). Therefore, after 120 min staining, a large amount of DSAI remained in cytoplasm, while DOX and small amount of DSAI entered nucleus. To further confirm this phenomenon, 7721 cells were stained with the GO-based DNA nanomaterial for only $30 \mathrm{~min}$, then the cells were washed with PBS buffer to eliminate the redundant dye. After washing, the cells were continuously incubated with fresh medium for another $0-90$ min to analyze the location of DSAI and DOX. Similar results came out compared to the results in Figure 2, further confirming the transfer route of DSAI and DOX in cells (Figure S4). In contrast, non-target cells (HepG2 and LO2) showed no emission or rather weak emission after stained with the GO-based DNA nanomaterial (Figure S5, S6), indicating that the GO-based DNA nanomaterial could hardly stain the non-target cells. Other non-target cells (such as 4T1, Hela, 3T3 cells) were also estimated, and none of these cells were labeled (Figure S7), solidly verifying the high labeling selectivity of GO-based DNA nanomaterial to target 7721 cells. 


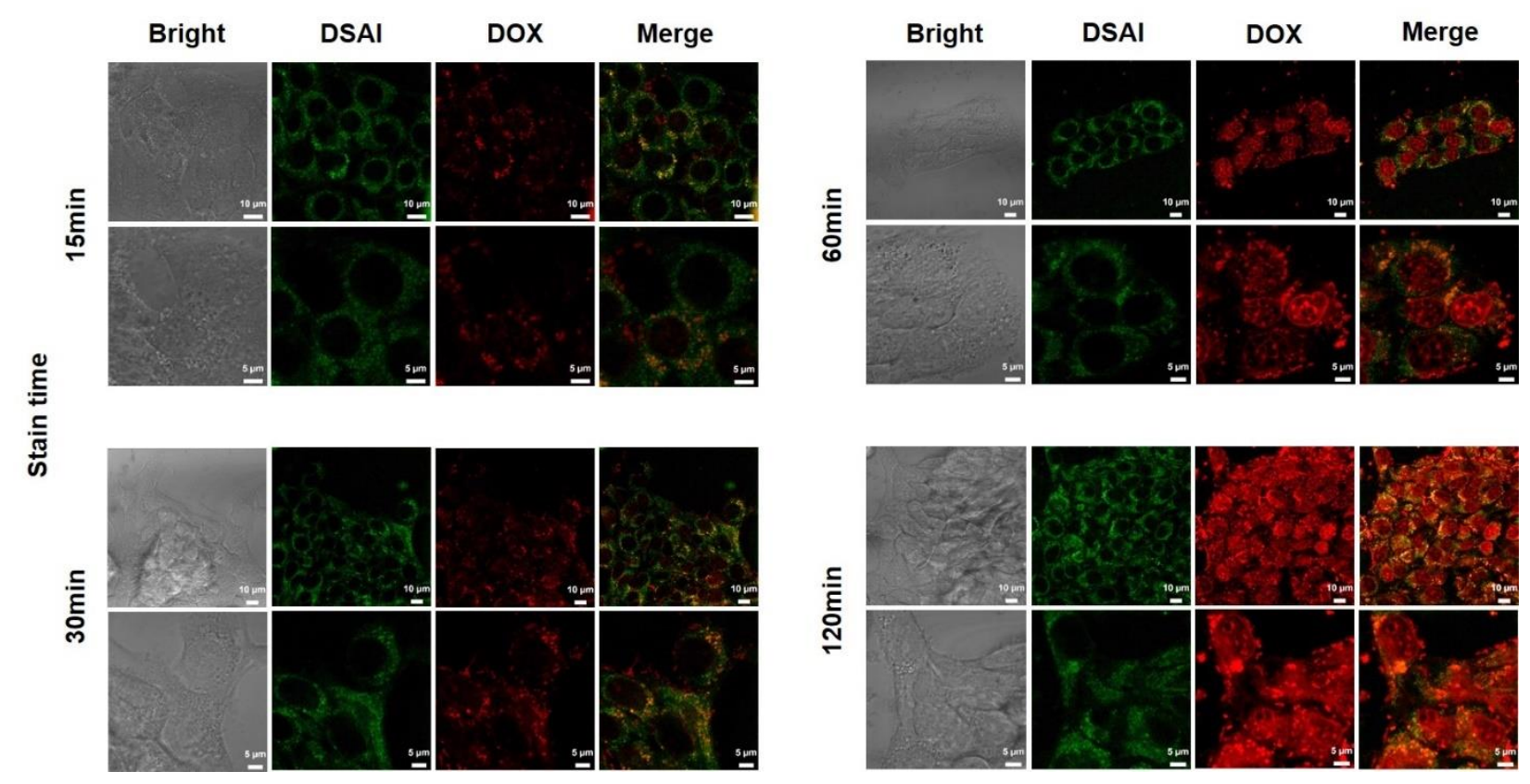

Figure 2. Confocal images (upper) and the relative magnifying images (lower) of 7721 cells after stained with GO-based DNA nanomaterial for different stain time. GO-based DNA nanomaterial: $1.0 \mu \mathrm{M}$ DSAI, $20 \mu \mathrm{M}$ DOX, 100 nM DNA-3, $40 \mu \mathrm{g} / \mathrm{mL}$ GO; Excitation: 405 nm, DSAI emission (green): 450 - $525 \mathrm{~nm}$; DOX emission (red): 530 - $740 \mathrm{~nm}$; Scale bar: $10 \mu \mathrm{m}$ for the integral images, $5 \mu \mathrm{m}$ for the magnifying images.

To analyze the selectivity for target cell and non-target cells without and with the aid of GO, the DNA nanomaterial was applied to stain the 7721, HepG2 and LO2 cells with different stain time. As shown in Figure S8 - S10, all the target and non-target cells were labeled with DNA nanomaterial, and DOX began to enter the nucleus within a short stain time (30 min). The mean fluorescence intensity of DSAI and DOX was concluded in Figure S11. In the absence of GO, the emission intensity of DSAI and DOX in HepG2, LO2 and 7721 cells was high, thus the comparison between non-target cells and target cell was insufficient. In the presence of GO, the emission of DSAI and DOX in non-target cells was rather weak, and the contrast to target cell 
was adequate. It indicated that the selectivity of DNA nanomaterial for target cell was greatly enhanced with the aid of GO.

After the selectivity verification to target 7721 cells, this GO-based DNA nanomaterial was also applied in microfluidic chip ${ }^{25-26}$ for target cell selection. The structure of the microfluidic chip was shown in Figure 3A, and its morphology image was shown in Figure 3B. The design of the microfluidic chip was according to the previous report ${ }^{27}$, which had a high capture efficiency for target cells. The channel of the microfluidic chip was a matrix with many pillars. The total length of channel was $5 \mathrm{~mm}$, and the width was $0.62 \mathrm{~mm}$. The pillar diameter and pillar height were two important factors for capture efficiency. The distance between two adjacent pillars were $50 \mu \mathrm{m}$, and the pillar diameter and height were $50 \mu \mathrm{m}$ and $11 \mu \mathrm{m}$, respectively. The height of channel was $30 \mu \mathrm{m}$, which was high enough to realize the cell float. The channel surface (including the pillar surface) used in this experiment was modified with the GO-based DNA nanomaterial. Three different cells (HepG2 cells, LO2 cells, 7721 cells) were injected in the channel at a speed of $100 \mu \mathrm{L} / \mathrm{h}$ (total volume: $100 \mu \mathrm{L}$, injection time: $1 \mathrm{~h}$ ). Then these three cells were transferred to cell dishes and incubated for $0.5 \mathrm{~h}$ to let the cells stick to the bottom. Among three different cells, only 7721 cells were labeled with DNA nanomaterials and presented red emission (Figure 3C). Both HepG2 cells and LO2 cells were unlabeled and had no emission. Therefore, this modified microfluidic chip had great potential in target cell screening technology. 
A

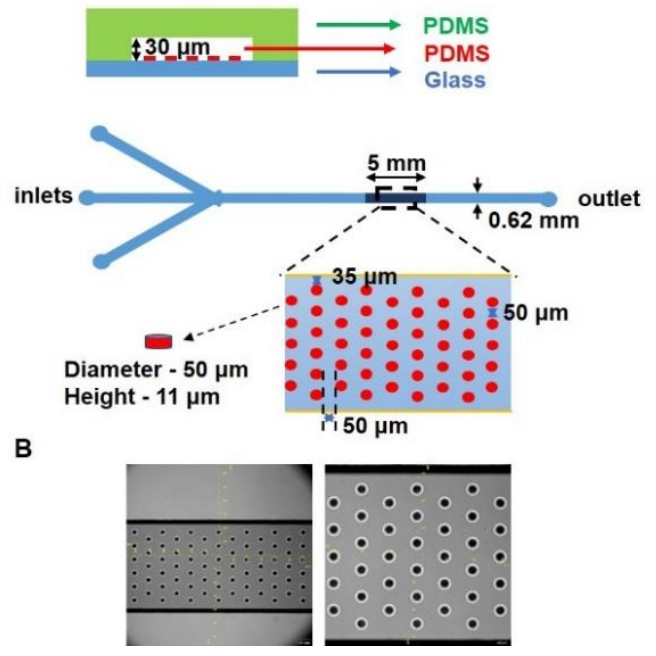

C

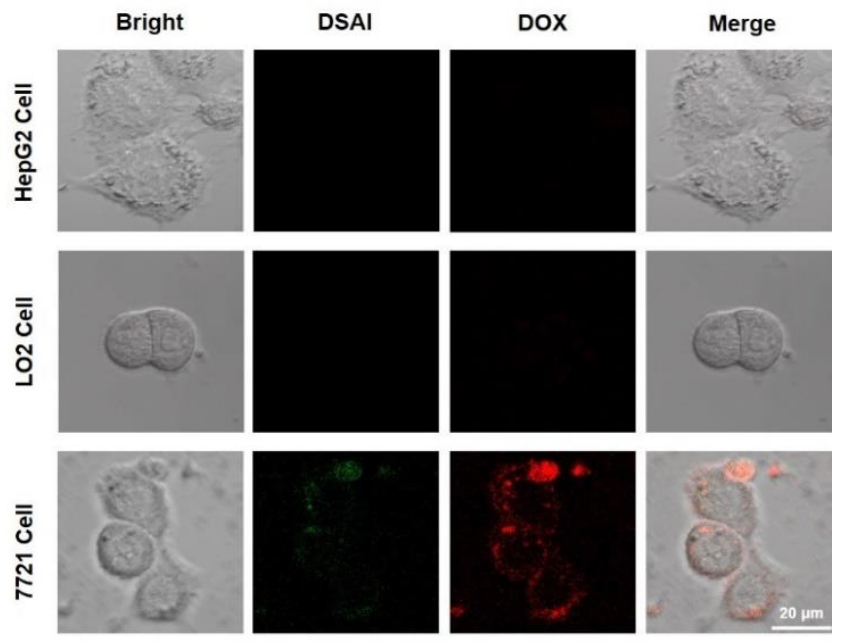

Figure 3. (A) The structure of the microfluidic chip. (B) The morphology image of the microfluidic chip by microscope imaging. (C) The Confocal images of HepG2 cells, LO2 cells and 7721 cells after the float through the microfluidic chip; Scale bar: $20 \mu \mathrm{m}$.

Finally, in vivo imaging and tumor treatment of the GO-based DNA nanomaterial were investigated. For in vivo imaging, the tumor was efficiently stained with the GO-based DNA nanomaterial after the intravenous injection within $24 \mathrm{~h}$ (Figure 4A). At $3 \mathrm{~h}$, intensive fluorescence signal was observed in the tumor section, indicating that the GO-based DNA nanomaterials presented a prominent accumulation in tumor region. With the prolonger time, the fluorescence signal underwent a gradual rise within $12 \mathrm{~h}$, then slowly declined due to the metabolism. However, the dissected tumor tissue remained a strong red emission after $24 \mathrm{~h}$ staining (Figure 4B). For in vivo treatment, the mice bearing tumor were intravenous injected with PBS, DNA/DSAI/GO and DNA/DSAI/GO/DOX (GO-based DNA nanomaterial), respectively. The changes of tumor volume after the 20-day treatment were recorded. As displayed in Figure 4D, the DNA/DSAI/GO/DOX group had an obvious inhibition on tumor growth during the treatment. Meanwhile, mice administrated with PBS or DNA/DSAI/GO 
served as the controls. In contrast, the tumor volumes in these control groups increased rapidly after the treatment. DNA/DSAI/GO group has a slight inhibition on tumor growth compared to PBS group, but this inhibition was extremely weaker than that of DNA/DSAI/GO/DOX group, illustrating that PBS and DNA/DSAI/GO could not sufficiently inhibit tumor growth. The similar result of tumor weight change appeared in Figure S12, the lightest tumor weight appeared in DNA/DSAI/GO/DOX group at day 20 after treatment, which was consistent with the tumor volume change. Afterwards, the in vivo immunohistochemical studies were further analyzed (Figure 4C). KI67 immuno-fluorescence staining of tumor slices verified that few proliferating tumor cells appeared after treated with DNA/DSAI/GO/DOX group, comparing with the excess and high-density tumor cells in the control groups. The TUNEL immuno-fluorescence staining of tumor slices further testified the serious cell apoptosis after treated with DNA/DSAI/GO/DOX group. By comparison, there were few apoptosis tumor cells after the treatment with the control groups. These data clearly illustrated that the GO-based DNA nanomaterials could be used as highly versatile theranostic agents for tumor imaging and therapy in vivo.

Considering the significance of therapeutic safety for nanomaterials, the potential systemic cytotoxicity was carefully estimated. As shown in Figure 4E, the body weights of all the treated mice were measured during the 20-day treatment with different groups, and there was no significant weight change, which indicated great therapeutic agent safety of the GO-based DNA nanomaterials. In addition, histological and hematological analyses were measured to figure out the biocompatibility. ${ }^{28}$ It was demonstrated that 30-day treatment with DNA/DSAI/GO/DOX group had no influence for major organs (Figure S13A) and blood biochemistry indexes (Figure S13B) compared to the control groups. These results verified the great imaging and therapeutic effects of GO-based DNA nanomaterial with good biocompatibility for in vivo applications. 
A

B

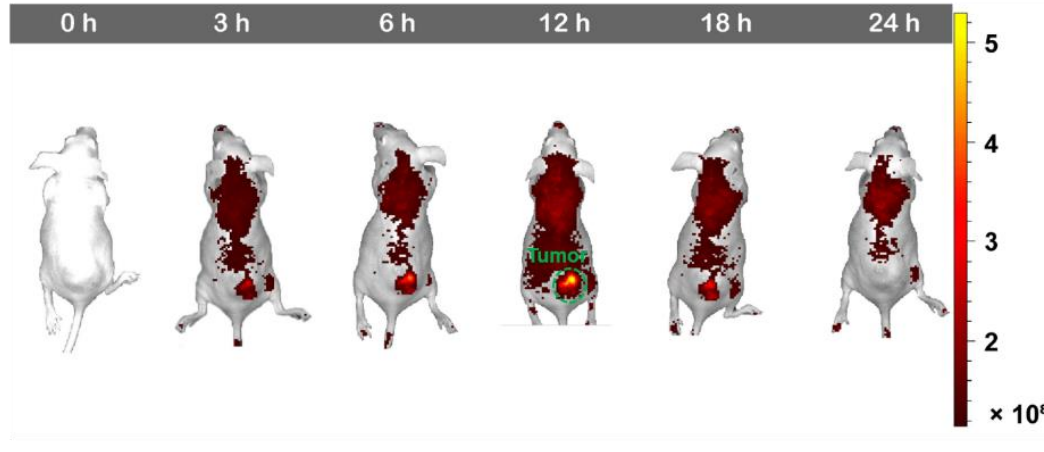

C
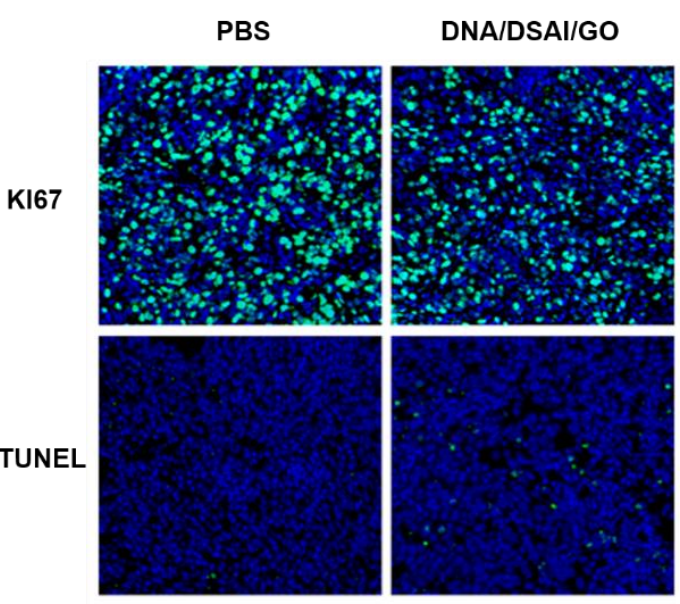

DNA/DSAI/GO/DOX

D
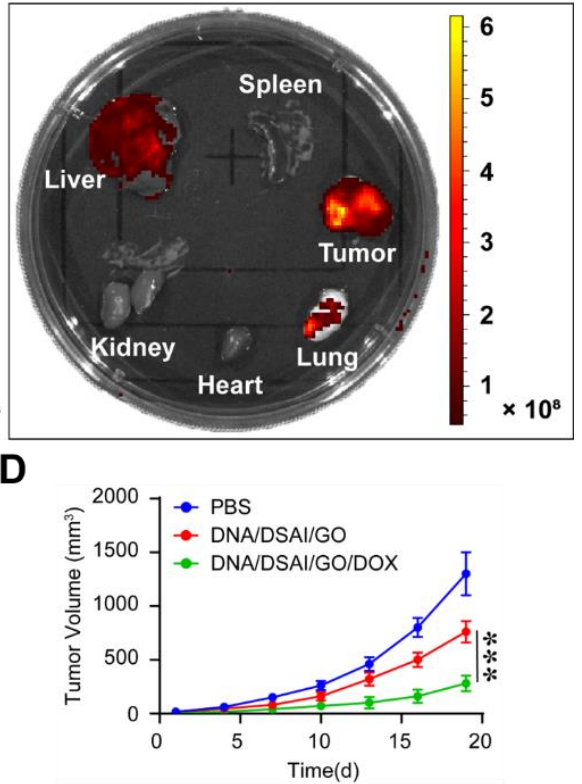

E

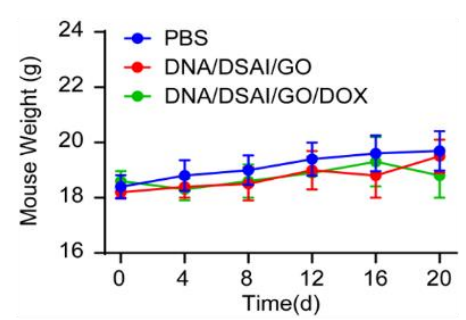

Figure 4. (A) In vivo imaging after the injection with the GO-based DNA nanomaterial for 0-24 h; (B) Organ imaging after the injection with GO-based DNA nanomaterial for $24 \mathrm{~h}$; (C) KI67 and TUNEL staining assays of tumor tissues after different treatment; Scale bar: $100 \mu \mathrm{m}$; (D) Tumor volume and (E) body weight changes of mice after different treatments for 20 days $(* * * \mathrm{p}$ $<0.001, \mathrm{n}=6)$.

\section{CONCLUSIONS}

In summary, we report a GO-based DNA nanomaterial for both in vitro and in vivo cancer diagnosis and therapy. Benefiting from the DNA self-assemble process, the DNA nanomaterial containing DNA tetrahedron structure and DNA aptamers acts as a great carrier, which can load both AIEgen and antitumor drug. Thanks to the good biocompatibility, high detection specificity 
to target liver tumor cell, and effective antitumor efficiency, both in vitro and in vivo results revealed that the presented nanomaterial is promising alternative for cancer theranostics. Our findings in this study may open new perspectives in the design of versatile carrier for target cell screening and treatment.

\section{ASSOCIATED CONTENT}

\section{Supporting Information.}

Additional experimental details; figures showing DNA-tetra characteristics, DLS measurement, confocal images, mean fluorescent intensity of the relative confocal images, histological and hematological data.

The Supporting Information is available free of charge via the Internet at http://pubs.acs.org.

\section{AUTHOR INFORMATION}

\section{Corresponding Author}

*E-mail: wliu@,whu.edu.cn

*E-mail: wangd@szu.edu.cn

*E-mail: tangbenz@ust.hk

\section{Author Contributions}

The manuscript was written through contributions of all authors. All authors have given approval to the final version of the manuscript. $\$$ These authors contributed equally.

\section{Notes}


The authors declare no competing financial interest.

\section{ACKNOWLEDGMENT}

This work was funded by the Developmental Fund for Science and Technology of Shenzhen government (Grant No. JCYJ20190808153415062, JCYJ20190808121417291), the Natural Science Foundation for Distinguished Young Scholars of Guangdong Province (2020B1515020011), the Natural Science Foundation of China (21801169). The authors also acknowledge the Instrumental Analysis Center of Shenzhen University. All the animal procedures were performed according to the guidelines of the Institutional Animal Care and Use Committee at Wuhan University.

\section{ABBREVIATIONS}

GO, graphene oxide; DOX, doxorubicin; AIEgens, aggregation-induced emission luminogens; FRET, Förster resonance energy transfer.

\section{REFERENCES}

(1) Liu, X.-Q.; Aizen, R.; Freeman, R.; Yehezkeli, O.; Willner, O. Multiplexed Aptasensors and Amplified DNA Sensors Using Functionalized Graphene Oxide: Application for Logic Gate Operations. ACS Nano 2012, 6, 3553-3563.

(2) Liu, X.-Q.; Wang, F.; Aizen, R.; Yehezkeli, O.; Willner, I. Graphene Oxide/Nucleic-AcidStabilized Silver Nanoclusters: Functional Hybrid Materials for Optical Aptamer Sensing and Multiplexed Analysis of Pathogenic DNAs. J. Am. Chem. Soc. 2013, 135, 11832-11839.

(3) He, X.-P.; Zang, Y.; James, T. D.; Li, J.; Chen, G.-R. Probing Disease-Related Proteins with Fluorogenic Composite Materials. Chem. Soc. Rev. 2015, 44, 4239-4248. 
(4) Ma, K.; Zhang, F.-L.; Sayyadi, N.; Chen, W.-J.; Anwer, A. G.; Care, A.; Xu, B.; Tian, W.J.; Goldys, E. M.; Liu, G.-Z. Turn-on Fluorescent Aptasensor Based on AIEgen Labeling for the Localization of IFN- $\gamma$ in Live Cells. ACS Sensors 2018, 3, 320-326.

(5) Ma, K.; Wang, H.; Li, H.-L.; Wang, S.; Li, X.; Xu, B.; Tian, W.-J. A Label-Free Aptasensor for Turn-on Fluorescent Detection of ATP Based on AIE-Active Probe and WaterSoluble Carbon Nanotubes. Sensor. Actuat. B-Chem. 2016, 230, 556-558.

(6) Ma, K.; Wang, H.; Li, H.-L.; Xu, B.; Tian, W.-J. Label-Free Detection for SNP Using AIE Probes and Carbon Nanotubes. Sensor. Actuat. B-Chem. 2017, 253, 92-96.

(7) Landry, M.-P.; Ando, H.; Chen, A.-Y.; Cao, J.-C.; Kottadiel, V.-I.; Chio, L.; Yang, D.; Dong, J.-Y.; Lu, T.-K.; Strano, M.-S. Single-Molecule Detection of Protein Efflux from Microorganisms Using Fluorescent Single-Walled Carbon Nanotube Sensor Arrays. Nat. Nanotech. 2017, 12, 368-377.

(8) Li, F.; Pei, H.; Wang, L.-H.; Liu, J.-X.; Gao, J.-M..; Jiang, B.-W.; Zhao, X.-C.; Fan, C.-H. Nanometrial-Based Fluorescent DNA Analysis: A Comparative Study of the Quenching Effect of Graphene Oxide, Carbon Nanotubes, and Gold Nanoparticles. Adv. Funct. Mater. 2013, 23, 4140-4148.

(9) Kim, T.; Park, J.-Y.; Hwang, J; Seo, G.; Kim, Y. Supramolecular Two-Dimensional Systems and Their Biological Applications. Adv. Mater. 2020, 32, 2002405.

(10) Yuan, H.-B.; Qi, J.-J.; Xing, C.-F.; An, H.-L.; Niu, R.-M.; Zhan, Y.; Fan, Y.-B.; Yan, W.M; Li, R.-H; Wang, B.; et al. Graphene-Oxide-Conjugated Polymer Hybrid Materials for Calmodulin Sensing by Using FRET Strategy. Adv. Funct. Mater. 2015, 25, 4412-4418. 
(11) de Avila, B.-E.-F.; Martin, A.; Soto, F.; Lopez-Ramirez, M.-A.; Campuzano, S.; VasquezMachado, G.-M.; Gao, W.-W.; Zhang, L.-F.; Wang, J. Single Cell Real-Time miRNAs Sensing Based on Nanomotors. ACS Nano. 2015, 9, 6756-6764.

(12) Jiang, Y.-J.; Wang, N.; Cheng, F.; Lin, H.-R.; Zhen, S.-J.; Li, Y.-F.; Li, C.-M.; Huang, C.Z. Dual Energy Transfer-Based DNA/Graphene Oxide Nanocomplex Probe for Highly Robust and Accurate Monitoring of Apoptosis-Related MicroRNAs. Anal. Chem. 2020, 92, 1156511572.

(13) Wang, Y.; Tang, L.-H.; Li, Z.-H.; Lin, Y.-H.; Li, J.-H. In Situ Simultaneous Monitoring of ATP and GTP Using a Graphene Oxide Nanosheet-Based Sensing Platform in Living Cells. Nat. Protoc. 2018, 8, 41143-41149.

(14) Tan, X.-H.; Chen, T.; Xiong, X.-L.; Mao, Y.; Zhu, G.-Z.; Yasun, E.; Li, C.-M.; Zhu, Z.; Tan, W.-H. Semiquantification of ATP in Live Cells Using Nonspecific Desorption of DNA from Graphene Oxide as the Internal Reference. Anal. Chem. 2012, 84, 8622-8627.

(15) Li, Z.-H.; Zhao, B.; Wang, D.-F.; Wen, Y.-L.; Liu, G.; Dong, H.-Q.; Song, S.-P.; Fan, C.H. DNA Nanostructure-Based Universal Microarray Platform for High-Efficiency Multiplex Bioanalysis in Biofluids. ACS Appl. Mater. Inter. 2014, 6, 17944-17953.

(16) Wang, S.; Liu, Z.-Y.; Tong, Y.; Zhai, Y.-Q.; Zhao, X.-J.; Yue, X.-M.; Qiao, Y.-Q.; Liu, Y.-H.; Yin, Y.-M.; Xi, R.-M.; et al. Improved Cancer Phototheranostic Efficacy of Hydrophobic IR780 via Parenteral Route by Association with Tetrahedral Nanostructured DNA. J. Control. Release 2021, 330, 483-492. 
(17) Liang, L.; Li, J.; Li, Q.; Huang, Q.; Shi, J.-Y.; Yan, H.; Fan, C.-H. Single-Particle Tracking and Modulation of Cell Entry Pathways of a Tetrahedral DNA Nanostructure in Live Cells. Angew. Chem. Int. Ed. 2014, 53, 7745-7750.

(18) Li, J.; Pei, H.; Zhu, B.; Liang, L.; Wei, M.; He, Y.; Chen, N.; Li, D.; Huang, Q.; Fan, C.H. Self-Assembled Multivalent DNA Nanostructures for Noninvasive Intracellular Delivery of Immunostimulatory CpG Oligonucleotides. ACS Nano 2011, 5, 8783-8789.

(19) Fan, D.-Q.; Wang, J.; Wang, E.-K.; Dong, S.-J. Propelling DNA Computing with Materials' Power: Recent Advancements in Innovative DNA Logic Computing System and Smart Bio-Applications. Adv. Sci. 2020, 7, 2001766.

(20) Zhou, Z.-X.; Sohn, Y.-S.; Nechushtai, R.; Willner, I. DNA Tetrahedra Modules as Versatile Optical Sensing Platforms for Multiplexed Analysis of miRNAs, Endonucleases, and Aptamer-Ligand Complexes. ACS Nano 2020, 14, 9021-9031.

(21) Qu, A.-H.; Wu, X.-L.; Li, S.; Sun, M.-Z.; Xu, L.-G.; Kuang, H.; Xu, C.-L. An NIRResponsive DNA-Mediated Nanotetrahedron Enhances the Clearance of Senscent Cells. $A d v$. Mater. 2020, 32, 2000184.

(22) Yan, D.-Y.; Wu, Q.; Wang, D.; Tang, B.-Z.; Innovative Synthetic Procedures for Luminogens Showing Aggregation-Induced Emission. Angew. Chem. Int. Edit. 2021, DOI: 10.1002/anie.202006191.

(23) Chen, N.-D.; Yang, X.-H.; Wang, Q.; Jian, L.-X.; Shi, H.; Qin, S.-Y.; Wang, K.-M.; Huang, J.; Liu, W.-J. Proof of Concept for Inhibiting Metastasis: Circulating Tumor Cell- 
Triggered Localized Release of Anticancer Agent via a Structure-Switching Aptamer. Chem. Commun. 2016, 52, 6789-6792.

(24) Li, X.; Ma, K.; Zhu, S.-J.; Yao, S.-Y.; Liu, Z.-Y.; Xu, B.; Yang, B.; Tian, W.-J. Fluorescent Aptasensor Based on Aggregation-Induced Emission Probe and Graphene Oxide. Anal. Chem. 2014, 86, 298-303.

(25) Niu, Z.-Q.; Kozminsky, M.-A.; Day, K.-C.; Palmbos, P.-L.; Day, M.-L.; Nagrath, S. Isolation and Characterization of Circulating Tumor Cells (CTCs) from Bladder Cancer Patients Using a Highly Sensitive Graphene Oxide-Based Microfluidic device (GO Chip). Clin. Cancer Res. 2020, 26, 86-86.

(26) Wu, Y.-H.; Okesola, B.-O.; Xu, J.; Korotkin, I.; Berardo, A.; Corridori, I.; di Brocchetti, F.-L.-P.; Kanczler, J.; Feng, J.-Y.; Li, W.-Q.; et al. Disordered Protein-Graphene Oxide Coassembly and Supramolecular Biofabrication of Functional Fluidic Devices. Nat. Commun. 2020, $11,1182$.

(27) Yu, X.-L.; He, R.-X.; Li, S.-S.; Cai, B.; Zhao, L.-B.; Liao, L.; Liu, W.; Zeng, Q.; Wang, H.; Guo, S.-S.; et al. Magneto-Controllable Capture and Release of Cancer Cells by Using a Micropillar Device Decorated with Graphite Oxide-Coated Magnetic Nanoparticles. Small 2013, 9, 3895-3901.

(28) Xie, W.; Deng, W.-W.; Zan, M.; Rao, L.; Yu, G.-T.; Zhu, D.-M.; Wu, W.-T.; Chen, B.; Ji, W.; Chen, L.; et al. Cancer Cell Membrane Camouflaged Nanoparticles to Realize Starvation Therapy Together with Checkpoint Blockades for Enhancing Cancer Therapy. ACS Nano 2019, $13,2849-2857$. 
